\title{
Evaluation of Airframe Noise Reduction Concepts via Simulations Using a Lattice Boltzmann Approach
}

\author{
Ehab Fares, ${ }^{*}$ Damiano Casalino, $\dagger$ \\ Exa GmbH, Curiestrasse 4, D-70563 Stuttgart, Germany \\ and \\ Mehdi R. Khorrami \\ NASA Langley Research Center, Hampton, Virginia, 23681
}

\begin{abstract}
Unsteady computations are presented for a high-fidelity, $18 \%$ scale, semi-span Gulfstream aircraft model in landing configuration, i.e. flap deflected at $39^{\circ}$ and main landing gear deployed. The simulations employ the lattice Boltzmann solver PowerFLOW® to simultaneously capture the flow physics and acoustics in the near field. Sound propagation to the far field is obtained using a Ffowes Williams and Hawkings acoustic analogy approach. In addition to the baseline geometry, which was presented previously, various noise reduction concepts for the flap and main landing gear are simulated. In particular, care is taken to fully resolve the complex geometrical details associated with these concepts in order to capture the resulting intricate local flow field thus enabling accurate prediction of their acoustic behavior. To determine aeroacoustic performance, the farfield noise predicted with the concepts applied is compared to high-fidelity simulations of the untreated baseline configurations. To assess the accuracy of the computed results, the aerodynamic and aeroacoustic impact of the noise reduction concepts is evaluated numerically and compared to experimental results for the same model. The trends and effectiveness of the simulated noise reduction concepts compare well with measured values and demonstrate that the computational approach is capable of capturing the primary effects of the acoustic treatment on a full aircraft model.
\end{abstract}

\section{Introduction}

Airframe noise constitutes a major component of the total noise generated by contemporary civil aircraft, especially during landing when the engines are typically at low thrust settings. The continuous growth in air travel has led to an increase in community noise exposure, resulting in stricter airport regulations nationally and in some cases also regionally. Expected future noise restrictions will inevitably lead to the development of overall quieter aircraft designs. A considerable number of studies, both experimental and numerical, have been conducted during the past two decades ${ }^{1}$ to acquire a better understanding of the sources of airframe noise and to develop and advance noise reduction technologies. Flight tests and wind tunnel experiments targeting airframe noise sources have been instrumental in the development of component-level semi-empirical prediction tools ${ }^{2,3,4}$ such as NASA's Aircraft Noise Prediction Program (ANOPP). State-of-the-art numerical simulations addressing the combined flow and acoustic problems of isolated components using Computational Fluid Dynamics (CFD) and Computational Aeroacoustics (CAA) are summarized in various contributions to the workshop on Benchmark Problems for Airframe Noise Computations (BANC) and associated publications. ${ }^{5,6}$

High-fidelity unsteady numerical simulations of an $18 \%$ scale, semi-span model of a Gulfstream aircraft in landing configuration (flap deflected at $39^{\circ}$ with main landing gear deployed) were recently performed using a Detached Eddy Simulation (DES) approach within NASA's FUN3D unstructured compressible Navier-Stokes solver ${ }^{7}$ and a Lattice Boltzmann-Very Large Eddy Simulation (LB-VLES) approach within Exa Corporation's PowerFLOW® solver. ${ }^{8}$ The simulations, which combined multiple components of a complete aircraft including main landing gear interaction with the flap side edge of a high-lift system, compared well with measured quantities (steady and unsteady surface pressures and far field noise), demonstrating the general feasibility of both flow solvers to accurately simulate complex aeroacoustic interactions. Of the two approaches, the lattice Boltzmann algorithm provides a unique set of advantages

* Technical Director, Aerospace Applications, Senior Member AIAA

$\uparrow$ Technical Director, Aerospace Aeroacoustics, Member AIAA

+ Aerospace Engineer, Computational AeroSciences Branch, Associate Fellow AIAA 
in terms of computational efficiency, high frequency resolution and handling of very complex geometrical details, thus making it an ideal tool for simulating various noise reduction technologies and their effect on the overall aeroacoustic characteristics of the configuration.

The experimental dataset used in this paper to evaluate the accuracy of the simulations was acquired during aerodynamic and aeroacoustic wind tunnel tests of various noise reduction technologies conducted in the NASA Langley Research Center (LaRC) 14- by 22-Foot Subsonic Tunnel (14x22) during February-April of 2013.The 18\% scale semi-span model in landing configuration was used as the test bed. Several earlier papers document the steady ${ }^{9}$ and unsteady ${ }^{10}$ measurements of the baseline configuration as well as the aerodynamic ${ }^{11}$ and aeroacoustic ${ }^{12}$ performance of the noise reduction concepts.

The computational results presented in this paper were extracted from simulations predating the $201314 \times 22$ test. These earlier simulations (conducted in 2011) were used to evaluate and refine some of the flap and main gear noise reduction concepts developed by NASA and its industry partners prior to wind tunnel testing. As such, they were of sufficient resolution to capture the relevant primary noise reduction trends. The experience gained with the 2011 simulations allowed us to be more efficient during the development of subsequent computations (2013-2014) for a reduced number of concepts at slightly higher resolution.

\section{Baseline Model Geometry and Noise Reduction Concepts}

The baseline configuration used for the simulations is an $18 \%$ scale, semi-span, high-fidelity Gulfstream aircraft model. The geometry comprises a fuselage, wing, flap, flow-through nacelle, pylon, and main landing gear. A detailed description of this model is provided in Ref. 9. The noise reduction concepts presented in this paper represent only a subset of those that were simulated prior to testing on the semi-span model in the $14 \times 22$ tunnel. They correspond to some of the better performing concepts.

The baseline geometry and three of the flap noise reduction concepts applied at the side edges are presented in Fig. 1. The FLEXible Side-Edge Link (FLEXSEL) concept is an elastomeric structural-linkage that connects the flap side edge with the main wing edge within a very short flap span that scales with the local maximum flap thickness. ${ }^{12}$ The Reactive Orthotropic Lattice Diffuser (ROLD) concept consists of three independent sets of passages or perforations that are orthogonally aligned in all three directions. ${ }^{12}$ Passages from each direction intersect, thereby creating a three dimensional lattice structure that is orthotropic in nature. Finally, the Flap Edge Noise Reduction Fins (FENoRFins) design $^{12}$ is an array of densely packed comb like structures (fins) in a 3-D arrangement extending inward in the spanwise direction from the flap side edge. Geometrical variations of these concepts, such as different hole and fin sizes or shapes, were simulated also but will not be shown here. All three concepts were evaluated computationally with the treatment applied to both flap side edges of the semi-span model.

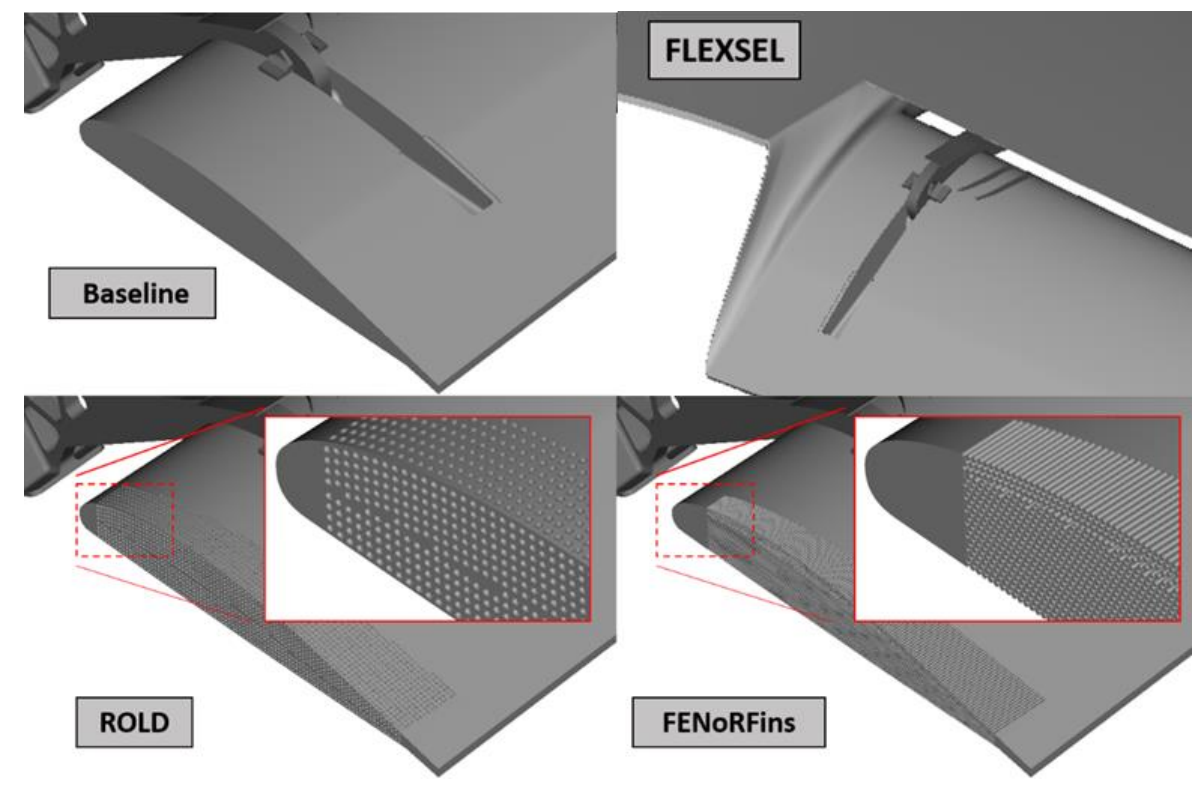

Figure 1. Schematic of flap edge noise reduction concepts applied to inboard edge 
The concepts for the main landing gear comprise an assortment of fairings that were tailored to reduce the noise from various components of the gear. In most cases, solid and porous versions of the fairings were designed and evaluated both computationally and experimentally on an isolated gear configuration. ${ }^{12}$ The effectiveness of the fairings was determined individually and as part of a larger collection of fairings applied to the gear. One of the best performing configurations consisted of several smaller fairings covering the upper segment of the gear plus porous fairings shielding the gear main posts and wheels; these are depicted in Fig. 2. Although not shown here, the present computational effort also contained a systematic evaluation of perforation size and spacing on the effectiveness of the porous knee fairing.

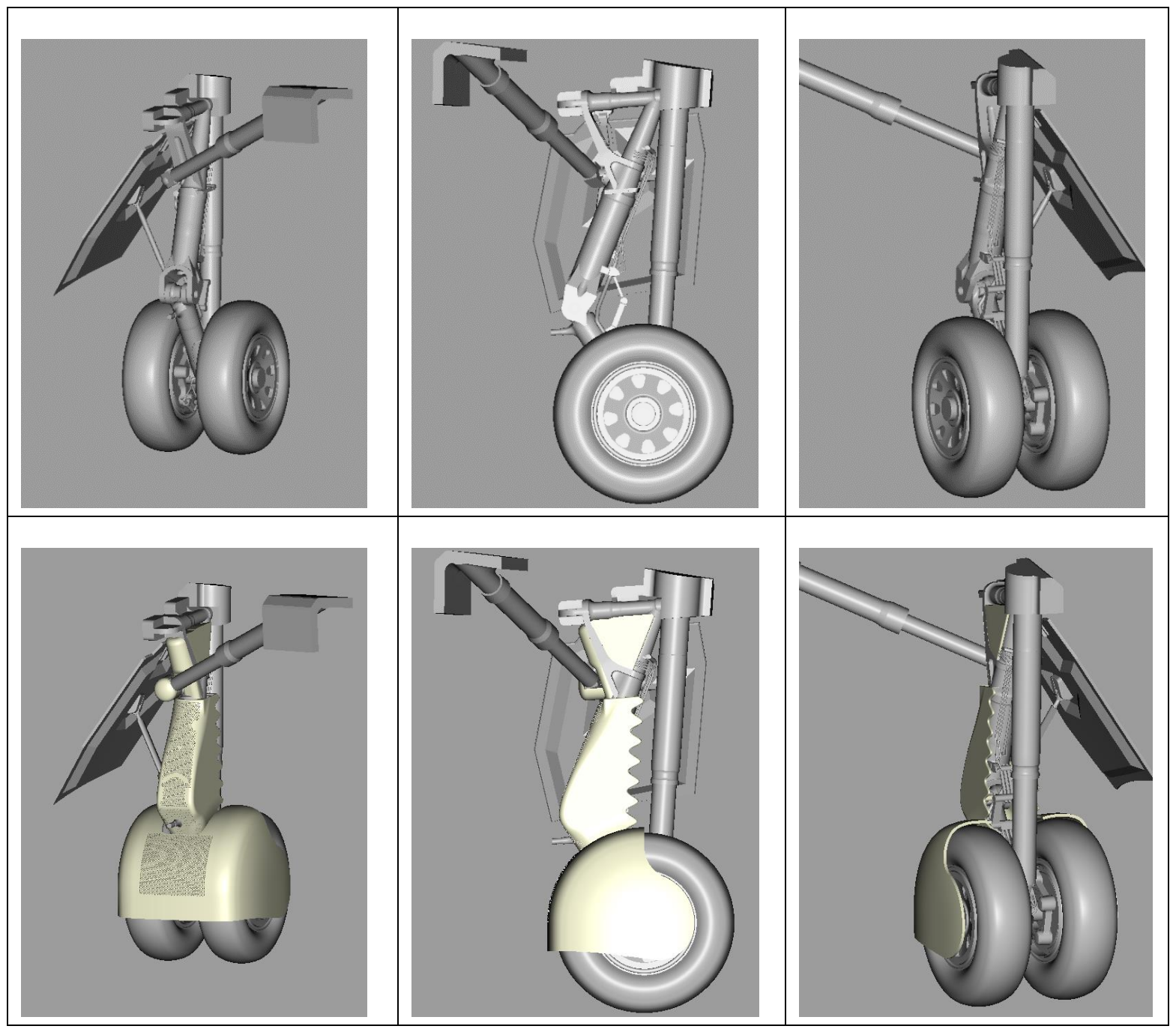

Figure 2. Isolated main landing gear geometry without (upper row) and with mounted noise reduction concepts (lower row)

The most promising combinations of flap noise reduction concepts and main landing gear fairings were simulated simultaneously on the $18 \%$ scale semi-span model with deployed flap and landing gear, as shown in Fig. 3. 


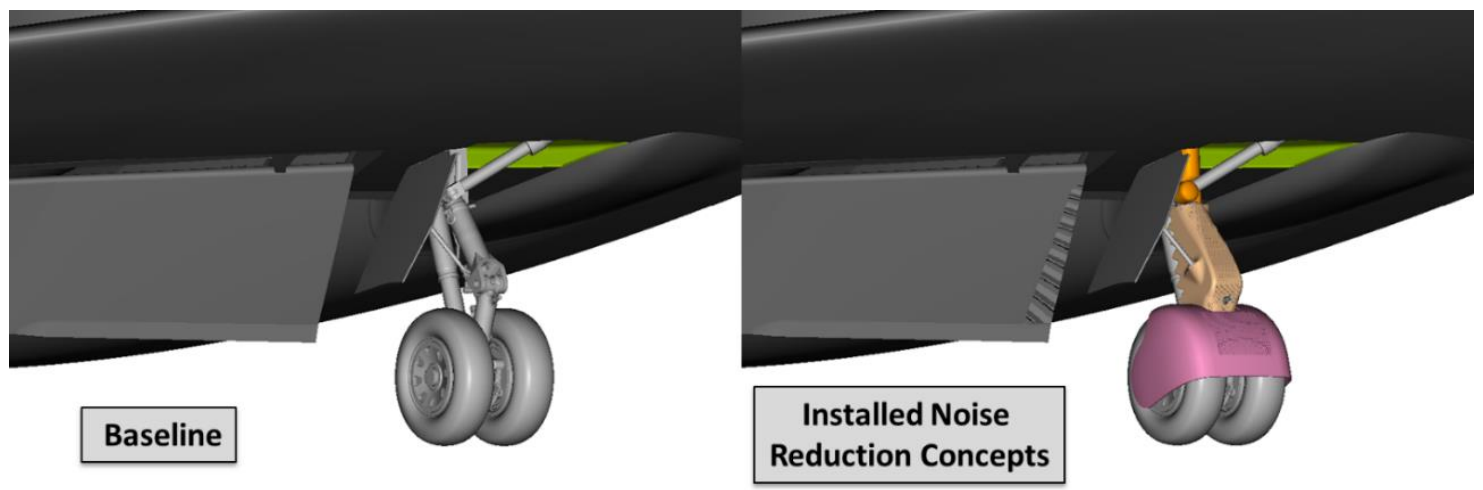

Figure 3. Semi-span aircraft model in landing configuration showing the inboard flap side edge and main landing gear geometries without and with noise reduction concepts installed.

\section{Computational Approach}

The numerical simulations were carried out using the commercial software PowerFLOW $®$, which is based on the three dimensional 19 state (D3Q19) lattice Boltzmann model (LBM). LBM is a CFD technology developed over the last 30 years ${ }^{13,14,15,16}$ and has been extensively validated for a wide variety of applications ranging from academic direct numerical simulation (DNS) cases to industrial flow problems in the fields of aerodynamics ${ }^{17}$ and aeroacoustics. ${ }^{8,18,19}$ In contrast to methods based on the Navier-Stokes (N-S) equations, LBM is based on a simpler and more general physics formulation. ${ }^{13}$ The motivation behind the approach is to simulate a fluid at a microscopic level where the physics are simpler and more general than the macroscopic continuum approach taken by the N-S equations. The LBM equations recover the macroscopic hydrodynamics of the Navier-Stokes equations ${ }^{17,20}$ through the Chapman-Enskog expansion. The simple, local formulation of the LBM equations allows a highly efficient implementation for distributed computations on thousands of processors. The low dissipation and dispersion properties of the numerical scheme typically produce aerodynamic and aeroacoustic results that are comparable to those obtained with classical CFD solvers that use higher-order large eddy simulation (LES), as shown theoretically in Refs. 22 and 23, and demonstrated in the comparative study of flow over tandem cylinders presented in Ref. 6.

The classical LBM scheme is typically valid in the low Mach number regime up to Mach 0.4. This method was applied for the work presented here. Recent extensions to the scheme ${ }^{24,25}$ however, allow the simulation of flows at higher Mach numbers as well.

\section{A. Turbulence Modelling}

The lattice Boltzmann flow simulation is equivalent to a Direct Numerical Simulation (DNS) of the flow. For high Reynolds number flows, such as those addressed in this work, turbulence modeling is incorporated into the lattice Boltzmann model by replacing the relaxation time, which is related to the thermodynamic chaotic motion of fluid particles, by a turbulent relaxation time that models the effect of turbulent chaotic motion on the statistics of fluid particle collisions ${ }^{26}$ The turbulent kinetic energy and the turbulent dissipation are obtained by solving a variant of the RNG k- $\varepsilon$ model. ${ }^{26}$ This model incorporates a swirl term that detects the occurrence of large-scale three-dimensional turbulent structures and reduces the influence of the turbulence model on the simulated flow accordingly. In this respect, the turbulent lattice Boltzmann flow model is analogous to a hybrid URANS-LES formulation, with the difference that, compared for instance to standard DES formulations, the blending between LES and URANS is based on the resolved turbulence structures and has therefore an adaptive character both in space and time. ${ }^{26}$ The swirl model together with the inherently unsteady nature of the Lattice-Boltzmann equation reproduce the large scale turbulent structures. This represents, from a pragmatic point of $v_{i e w^{27}}$, a key factor in predicting LES similar solutions on coarse grids through the use of an unsteady turbulence model, a methodology referred to as Very Large Eddy Simulation (VLES). This LBM-VLES based description of turbulent fluctuation carries flow history and upstream information, and contains high order terms to account for the nonlinearity of the Reynolds stress. ${ }^{28}$ This is in contrast to NavierStokes based methods, which generally use the conventional linear eddy viscosity based Reynolds stress closure models. 


\section{B. Wall Treatment}

The standard Lattice-Boltzmann bounce-back boundary condition for no-slip or the specular reflection for freeslip condition are generalized through a volumetric formulation ${ }^{13,14}$ near the wall for arbitrarily oriented surface elements (Surfels) within the Cartesian volume elements (Voxels). This formulation of the boundary condition on a curved surface cutting the Cartesian grid is automatically mass, momentum, and energy conservative while maintaining the general spatial second-order accuracy of the underlying LBM numerical scheme.

In order to reduce the resolution requirements near the wall for high Reynolds number flows, a hybrid wall function is used to model the region of the boundary layer closest to the solid surfaces. The wall function model described in Ref. 29 is an extension of the standard log law of the wall, including the laminar sub-layer and the effect of favorable and adverse pressure gradients. It accounts for surface roughness through a length parameter as well as the laminarto-turbulent transition of the boundary layer though a specified laminar region on the surface. Various validations of the wall model, especially the accurate prediction of the separation point on a smooth body such as stall of a wing, have been published previously. ${ }^{17,30}$

\section{Complex Geometry Handling and Meshing}

The lattice Boltzmann approach is solved on Cartesian meshes, which are generated automatically for any geometrically complex shape. Variable refinement regions (VR) can be defined to allow for local mesh refinement of the grid size by successive factors of two. The position and size of the local refinement regions used correspond to the baseline configuration documented previously in Ref. 8. Compared to the baseline configuration, the procedure to include noise reduction concept geometries consists of merely replacing the baseline component surface description with the modified geometry description and rerunning the automatic discretization based on the prescribed VR definition. This procedure basically eliminates the manual work associated with volume meshing and re-meshing for such modifications, independently of the level of geometrical detail required. The meshes change only locally and preserve the same off body and farfield volume mesh distribution and hence ensure a better, more accurate comparison between the baseline and modified configurations. An illustration of the level of geometrical detail resolved in the simulation is documented in Fig. 4. Additional mesh resolution studies were generally conducted for all the noise reduction concepts to confirm that relatively converged results had been attained. In this paper, however, only the finest mesh resolution simulated is reported.

\section{Computational Procedure}

Initial and boundary conditions as well as spatial and temporal resolution are all identical to the previously published baseline configuration simulation. ${ }^{8}$ An acoustic analogy approach based on the Ffowcs Williams and Hawkings (FWH) formulation ${ }^{32}$ was used to extrapolate the computed near-field fluctuations to the far field. The employed FWH formulation was based on the retarded-time formulation 1A by Farassat, ${ }^{33}$ extended to account for uniform mean flow convection effects to simulate the noise generated and measured in an ideal infinite wind tunnel. ${ }^{34}$ Integration was performed on different solid surfaces of the aircraft and on permeable surfaces surrounding different components of the aircraft. As shown in Ref. $8,{ }^{8}$ both solid and permeable integrations including the complete aircraft produce the same far field signature up to the cut-off frequency of the permeable surface, which is typically considerably lower than the solid one due to the coarser volumetric mesh in the off body region. In this paper only the FWH propagation based on the solid formulation will be reported. 

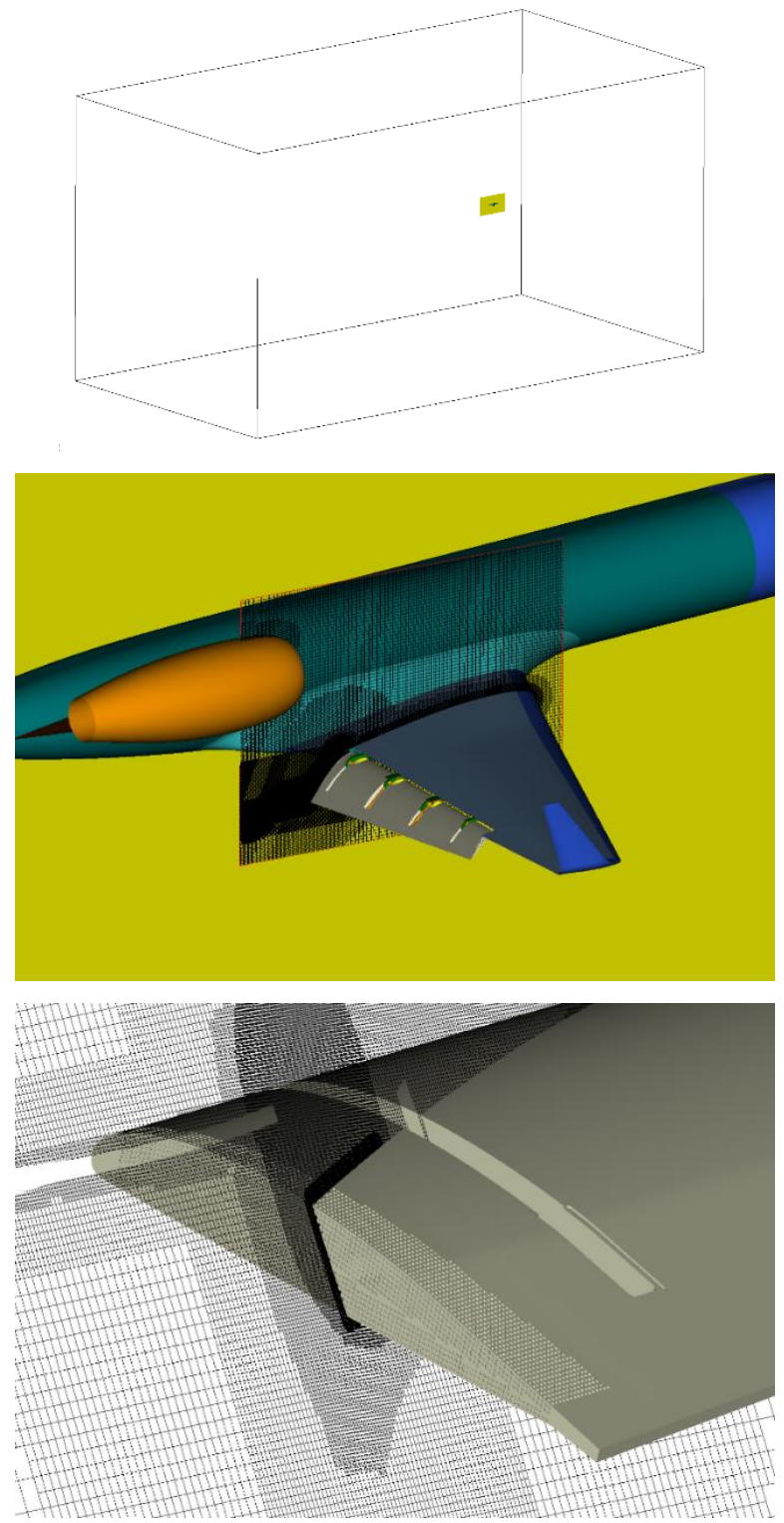
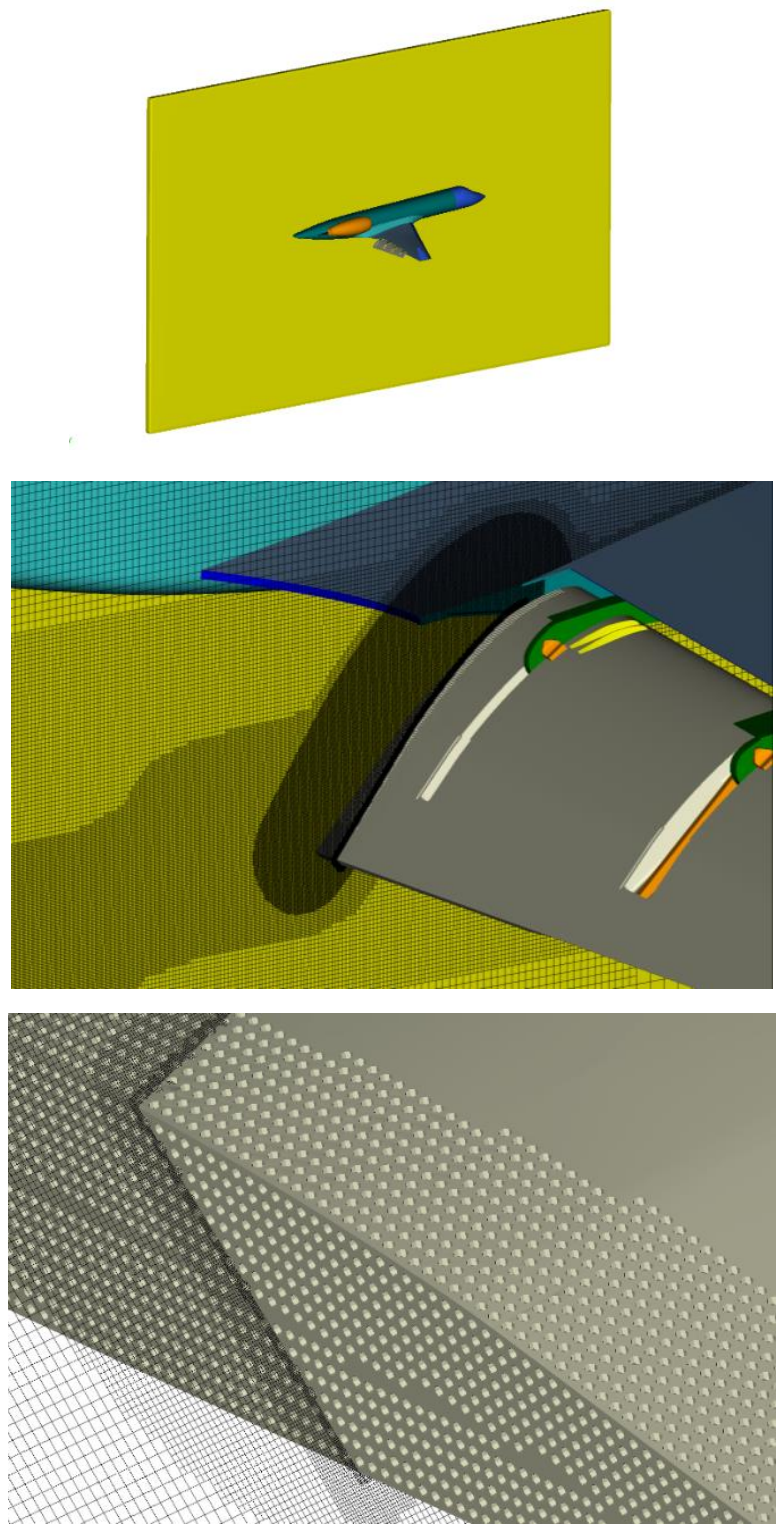

Figure 4. Simulation volume (top left) and successive closer views of geometry and mesh at the inboard flap side edge region (top right to bottom right)

\section{Results and Discussion}

\section{A. Global View}

Utilizing a Gulfstream aircraft as the test bed, acoustic measurements during the 2006 NASA-Gulfstream joint flight tes ${ }^{35}$ highlighted the flap sides and main landing gear as the more prominent airframe noise sources for landing configuration. Thus, the noise reduction effort targeted these two important airframe sources. The noise abatement concepts evaluated in this study were developed as part of NASA's Environmentally Responsible Aviation (ERA) project effort to mitigate aircraft noise without adversely impacting the aerodynamic efficiency of the aircraft. ${ }^{12}$ Flap and gear concept design philosophy, development, permutations, and corresponding acoustic measurements obtained in the $14 \times 22$ tunnel are provided in Ref. 12. The aerodynamic performance of the noise reduction concepts (presented in Ref. 11) revealed that very minor changes in the model lift coefficient $\left(C_{L}\right)$, mostly less than $0.5 \%$ and generally 
falling within the instrumentation and measurement uncertainty bounds, were incurred. As will be shown in the next section, minor changes in $\mathrm{C}_{\mathrm{L}}$ and drag coefficient $\left(\mathrm{C}_{\mathrm{D}}\right)$ were predicted reasonably well by the simulations.

The experimental and computational evaluation of the noise reduction concepts were achieved using two baseline configurations of the $18 \%$ scale model: a) flap deflected at $39^{\circ}$, gear removed and b) flap deflected at $39^{\circ}$, main gear deployed. The computed solutions were obtained for a representation of the open-jet $14 \times 22$ tunnel configuration (i.e., viscous flow on the floor and free-air in all directions above the floor), maintaining the same test conditions and model landing configurations as in the $14 \times 22$ tunnel setup. The simulations were conducted at an angle of attack (AOA) of $3^{\circ}$, Mach number $(\mathrm{M})$ of 0.20 , and Reynolds number $(\mathrm{Re})$ of $3.40 \times 10^{6}$ based on the mean aerodynamic chord of the $18 \%$ scale model matching the flow conditions of the $14 \times 22$ test.

\section{B. Flap Noise Reduction Concepts}

In this section, computational results are presented for the configuration with flap deflected $39^{\circ}$ and main gear removed. Simulation-based results for the three flap side edge noise reduction concepts considered here are summarized in Table 1. The Overall Sound Pressure Level (OASPL) is integrated over the model scale frequency range of $1 \mathrm{kHz}$ to $30 \mathrm{kHz}$ at an overhead microphone position 17.5 feet away from the model. Given our interest in the broadband component of airframe noise, the integrations were performed excluding a few prominent low frequency tonal peaks present in the computed spectra. Un-weighted and D-weighted OASPL values obtained from integrating the spectra between $1 \mathrm{kHz}$ and $30 \mathrm{kHz}$ model scale $(180 \mathrm{~Hz}$ and $5,400 \mathrm{~Hz}$ full scale) are shown. Dweighting produces results that closely track the noise reduction levels in PNdB. From Table 1, the achieved reduction in broadband noise ranges from $\sim 4$ to $5 \mathrm{~dB}$. The corresponding noise reduction levels measured during the $14 \times 22$ test are tabulated in Table 2. The measurements show reduction values in the range of $\sim 3$ to $4 \mathrm{~dB}$. Notice that all the concepts provided a similar amount of reduction. Note that in both tables positive values represent improvements over baseline values. As reported in Ref. 12, the spectra measured at mid- to high-frequencies show contamination from secondary noise sources caused by the close proximity of the instrumentation wiring to the middle two flap brackets. The added noise masked the true performance of the concepts at high frequencies. The two instrumentation bundles were absent in our simulations, explaining part of the differences between the computed and measured noise reduction levels for each concept. However, as clearly demonstrated in Ref. 12, the ROLD and FENoRFins concepts also suffer from self-generated noise. As will be shown later in this paper, this effect was not captured by the computations due to insufficient resolution. Additional parametric computational studies (e.g., hole size of the ROLD, fin diameter and spacing of the FENorFins) were performed, but will not be reported here. In general, most trends captured by the simulations were confirmed by the wind tunnel measurements.

Table 1. Simulated acoustic and aerodynamic performance for flap noise reduction concepts

\begin{tabular}{|c|c|c|c|}
\hline PowerFLOW Simulations & FLEXSEL & ROLD & FENoRFins \\
\hline$\Delta_{\text {(baseline - concept) }}$ OASPL [dB] $(1 \mathrm{kHz}-30 \mathrm{kHz})$ & 4.3 & 4.7 & 3.9 \\
\hline$\Delta_{\text {(baseline - concept) }}$ OASPL [dBD] $(1 \mathrm{kHz}-30 \mathrm{kHz})$ & 3.4 & 4.5 & 4.5 \\
\hline$\Delta_{\text {(concept - baseline) }} \mathrm{C}_{\mathrm{L}}[\%]$ & 1.70 & -0.70 & -0.40 \\
\hline$\Delta_{\text {(concept - baseline) }} C_{\mathrm{D}}[\%]$ & -1.19 & -1.31 & -1.34 \\
\hline$\Delta_{\text {(concept - baseline) }} \mathrm{E}[\%]$ & 2.90 & 0.60 & 0.90 \\
\hline
\end{tabular}


Table 2. Measured acoustic and aerodynamic performance for flap noise reduction concepts

\begin{tabular}{|c|c|c|c|}
\hline Experiment in $14 \times 22 \mathrm{WT}$ & FLEXSEL & ROLD* & FENoRFins* \\
\hline$\Delta_{\text {(baseline - concept) }}$ OASPL [dB] $(1 \mathrm{kHz}-30 \mathrm{kHz})$ & 3.6 & 3.9 & 3.5 \\
\hline$\Delta_{\text {(baseline - concept) }}$ OASPL [dBD] $\quad(1 \mathrm{kHz}-30 \mathrm{kHz})$ & 3.3 & 2.0 & 2.9 \\
\hline$\Delta_{\text {(concept - baseline) }} C_{\mathrm{L}}[\%]$ & 2.00 & -0.60 & -0.30 \\
\hline$\Delta_{\text {(concept - baseline) }} C_{\mathrm{D}}[\%]$ & -1.25 & -2.75 & -1.50 \\
\hline$\Delta_{\text {(concept - baseline) }} \mathrm{E}[\%]$ & 3.25 & 2.25 & 1.70 \\
\hline
\end{tabular}

*The aerodynamic values shown here correspond to the smallest holes and fin diameter tested

The changes in lift and drag caused by the application of the noise reduction concepts resulted in an overall improvement in efficiency, $\mathrm{E}=\mathrm{C}_{\mathrm{L}} / \mathrm{C}_{\mathrm{D}}$, ranging from $0.5 \%$ to $\sim 3 \%$ as compared to the baseline. Unlike FLEXSEL, note from Table 1 that both ROLD and FENoRFins show a slight lift degradation of $\leq 0.7 \%$, more or less falling within the computational uncertainties. In contrast, FLEXSEL produced a measurable level of lift improvement on the order of $1.5 \%$ to $2.0 \%$. These values, which were similar to the corresponding model force measurements from the $14 \times 22$ test ${ }^{11}$ are shown in Table 2.
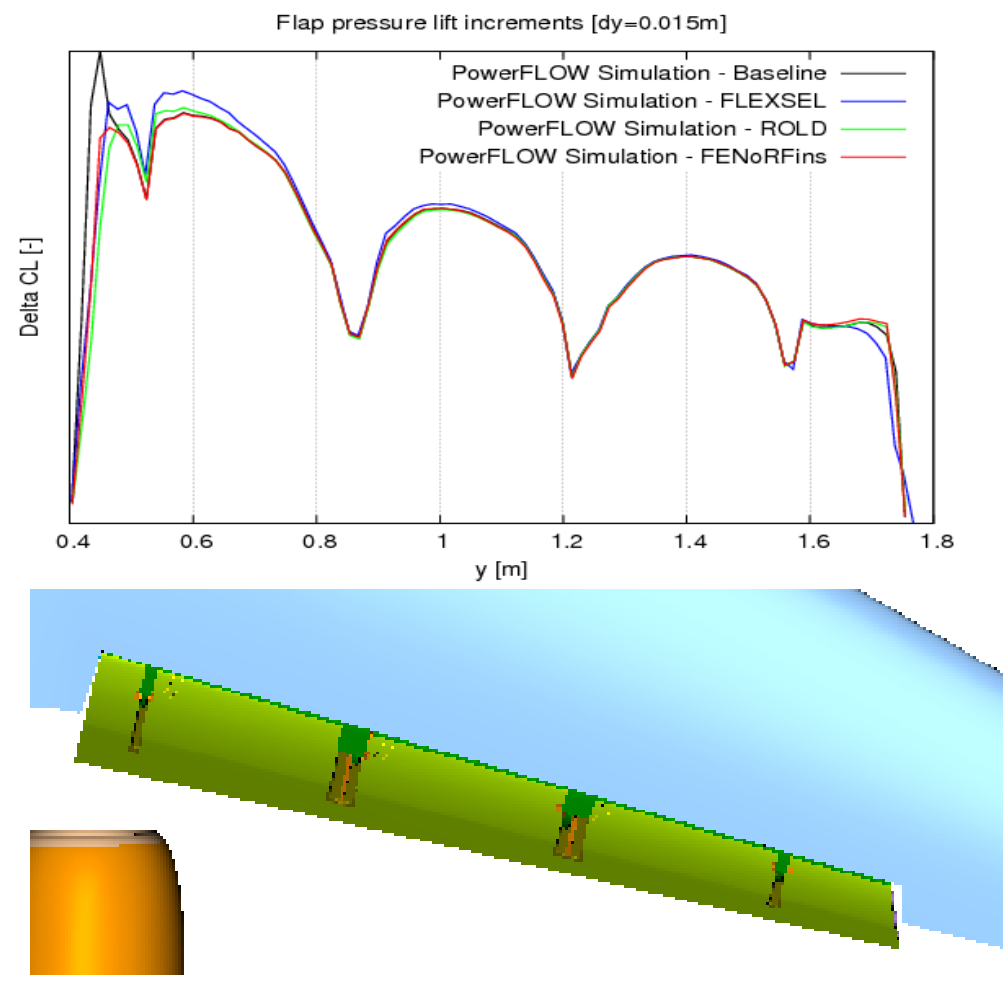

Figure 5. Flap lift spanwise distributions

A comparison of the flap lift distribution for the simulated configurations (Figure 1) is displayed in Figure 5 highlighting the modification of integrated local lift at the flap side edges by the installed concepts. Notice that the three concepts virtually eliminate the vortex-induced lift that is manifested as a sharp peak in the spanwise lift distribution at the flap inboard tip. The minor loss of lift associated with ROLD and FENoRFins, reported in Table 1, is presumably caused by this weakening of the tip vortex. The alteration of the spanwise lift distribution for these two 
concepts is generally confined to the inboard edge region with the rest of the flap remaining unaffected. In contrast, acting similarly to a fence, the FLEXSEL concept noticeably increases lift over $20 \%$ of the inboard section of the flap due to reduction in induced drag. Although there is a small loss of lift at the outboard tip, the overall lift produced by the FLEXSEL flap is higher than that of the baseline configuration. A closer look at the averaged inboard flap surface pressure distribution of the three concepts, compared to the baseline, clearly illustrates the reduction of the suction peak produced by the vortex core imprint on the surface (see Fig. 6). The largest reduction in peak suction pressure seems to be produced by the ROLD concept. FLEXSEL generates a different mechanism for altering vortex formation at the edge, which can be better seen in the planar cuts shown in Figure 7.

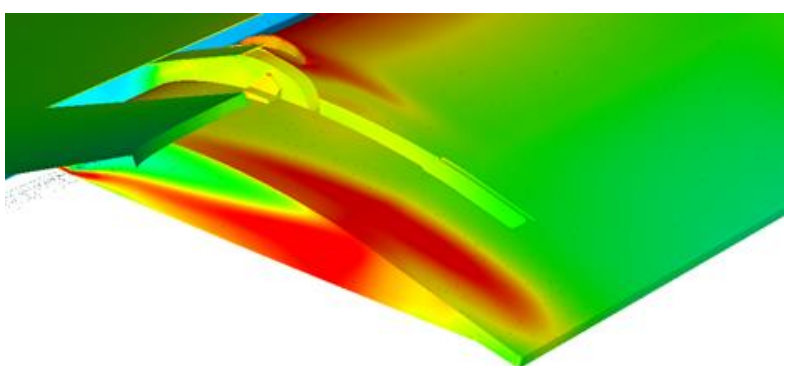

a) Baseline
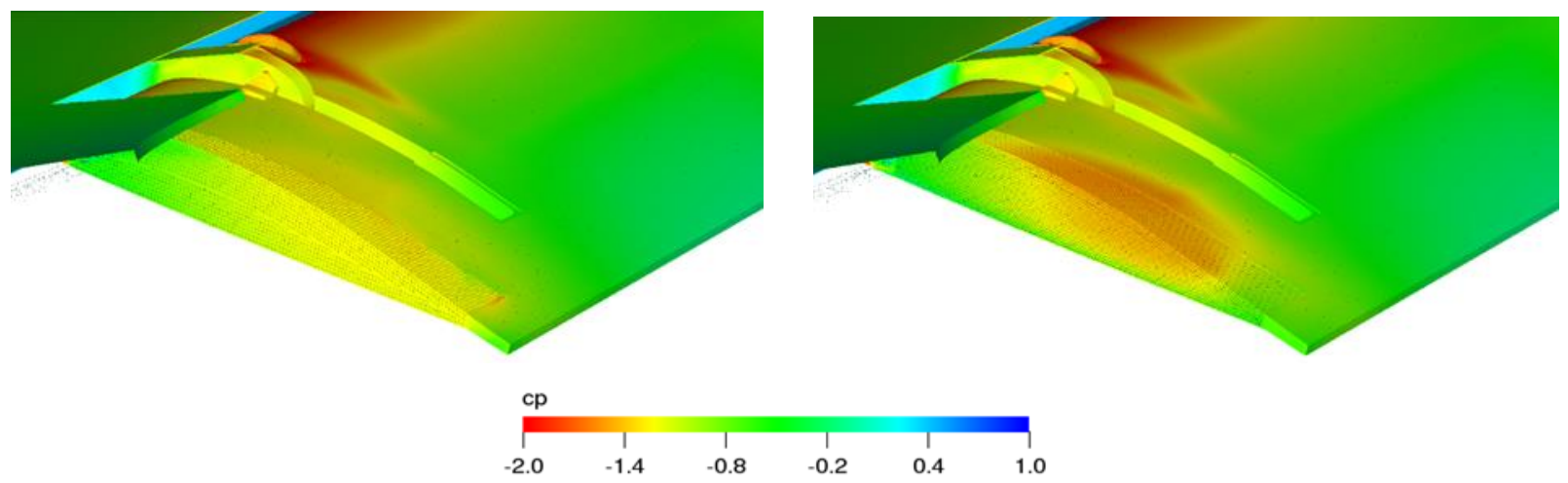

c) ROLD

d) FENoRFins

Figure 6. Surface pressure field at a the inboard flap side edge
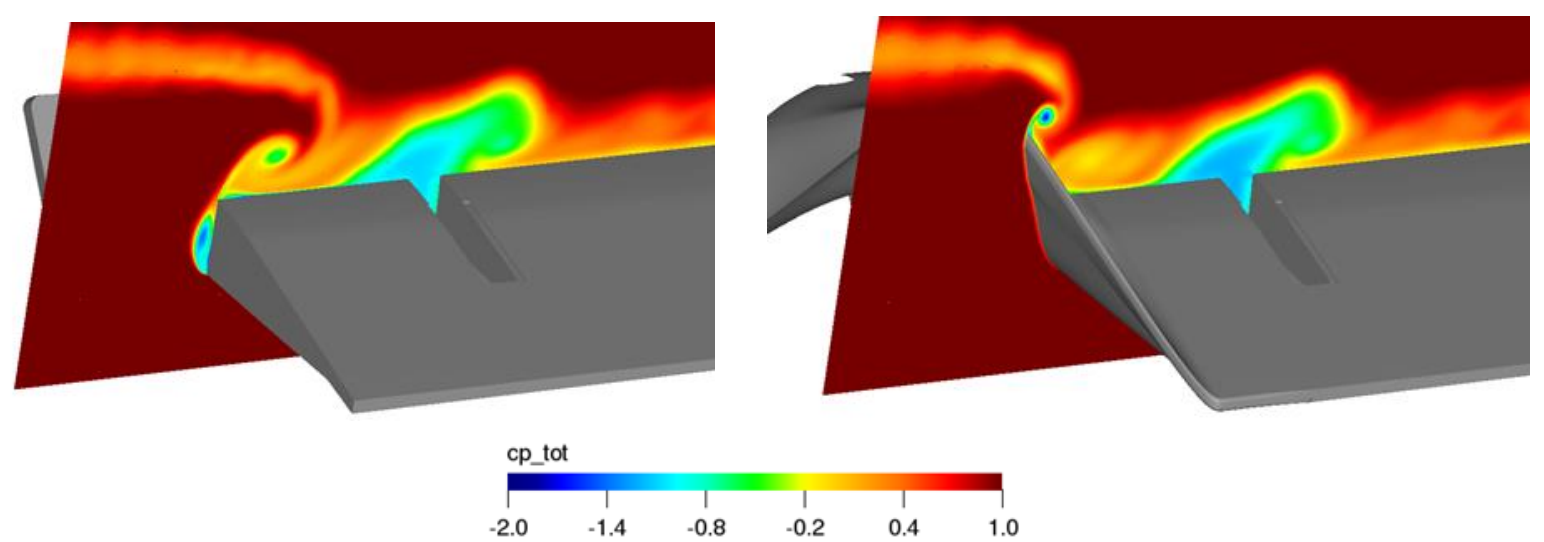

a) Baseline

b) FLEXEL

Figure 7. Averaged total pressure field at a section crossing the inboard flap side edge 


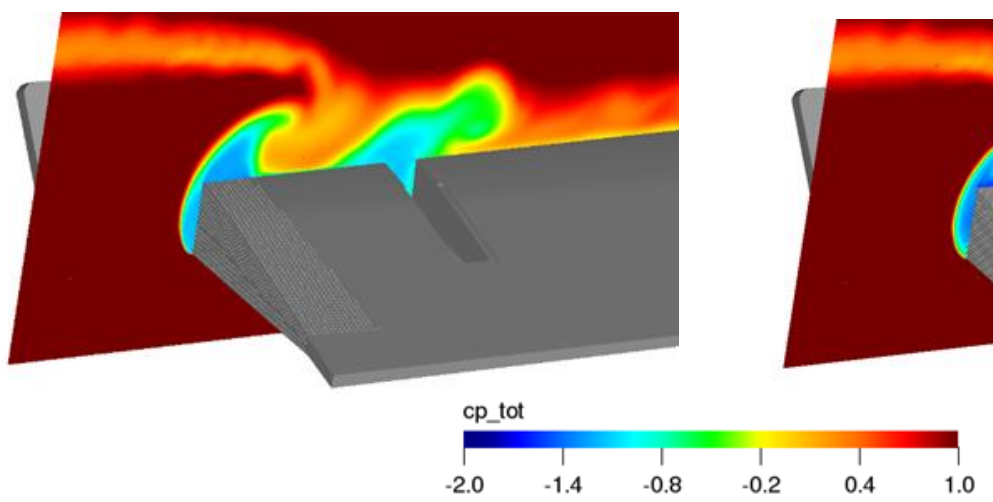

c) ROLD

d) FENoRFins

Figure 7. Concluded

Total pressure field at a section crossing the inboard flap side edge for the baseline, FLEXSEL, ROLD, and FENoRFins documented for the averaged (Fig.7) and instantaneous (Figure 8) flow field, confirming significant modifications of the tip flow structure by ROLD and, to a lesser extent, by FENoRFins. Acting like a fence, the FLEXSEL concept imposes a nearly total change of the tip flow features causing vortex formation and position to occur at larger distances above the flap surface. The changes observed in Figs. 7 and 8 tend to support our findings on the noise generation mechanisms at flap side edges. ${ }^{36}$ The analysis presented in Ref. 36 indicates that there exists a direct relationship between farfield noise and the lift fluctuations experienced at the tip caused by movement/oscillations of the coherent vortex system. Therefore, potentially effective noise reduction concepts must severely curtail vortex oscillation in addition to dampen the surface pressure fluctuations imposed by the turbulent flow field.

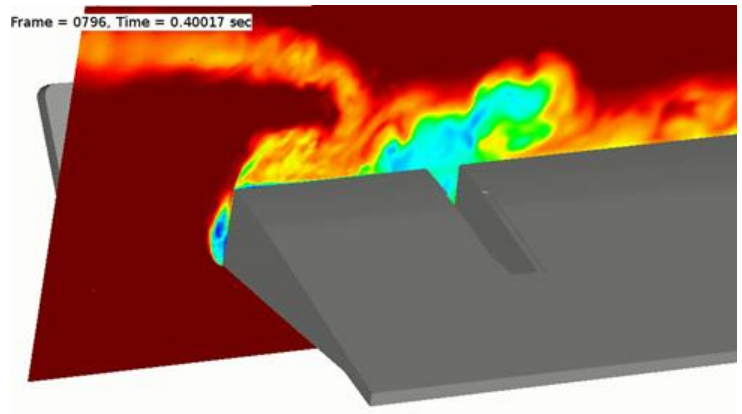

a) Baseline

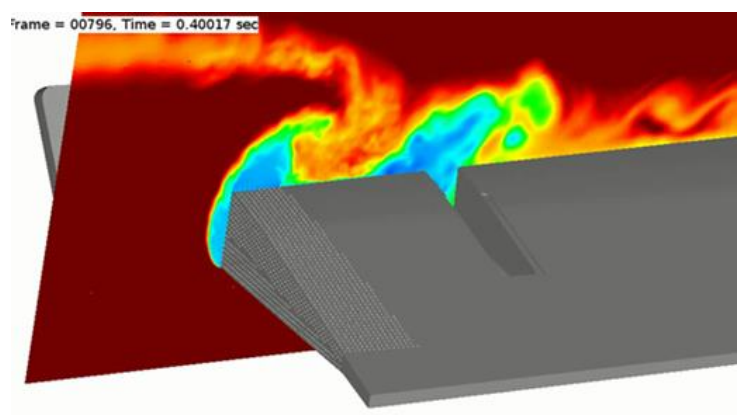

cp_tot

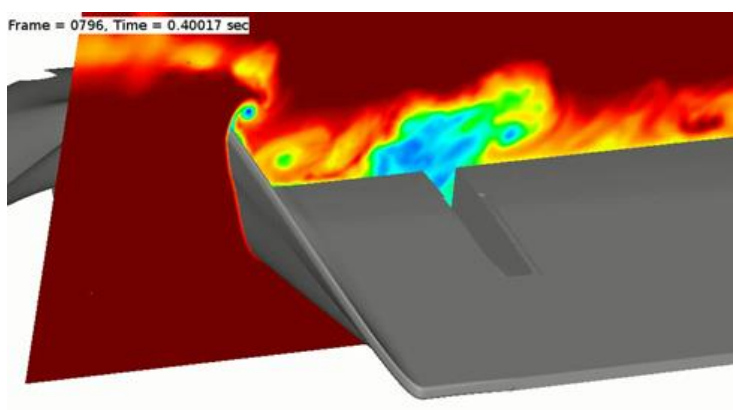

b) FLEXEL
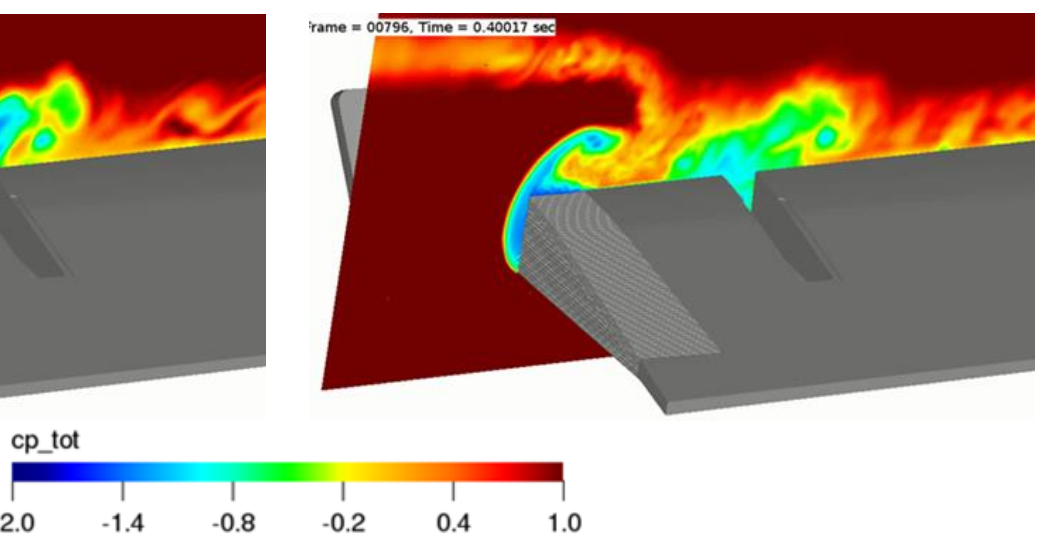

c) ROLD

d) FENoRFins

Figure 8. Instantaneous total pressure field at a section crossing the inboard flap side edge 
Simulated and measured farfield noise spectra for the baseline and the three flap concepts are plotted in Fig. 9. In this figure, the experimental Power Spectral Density (PSD) values were obtained by integrating the beamform maps generated from processing the microphone array output over an area that contained only the model wing and flap (see Ref. 12 for more details). This was done so to exclude the contribution from the noise sources that were generated near the region where the model abuts the wind tunnel floor. The simulated PSD values were obtained using the pressure field over the entire model surface. An alternate farfield noise calculation excluding contributions from the fuselage and nacelle produced nearly the same spectra, indicating that the noise sources residing at the flap tips are dominant.

For the baseline case (Fig. 9a), the broadband component of farfield noise is predicted rather well for most of the frequency range of interest. The broad tonal hump observed between $7 \mathrm{kHz}$ and $10 \mathrm{kHz}$ in the measured PSD is associated with tip vortex-cavity interaction at the flap outboard side edge. ${ }^{12}$ Vortex-cavity interaction was not captured by these earlier simulations because of insufficient spatial resolution. For the FLEXSEL and ROLD concepts (Figs. 9b and 9c), good agreement for the broadband component of noise was obtained for frequencies up to about 7 $\mathrm{kHz}$. Above this frequency, the measured spectrum displays higher noise levels caused by the noise reduction concept self-noise and contributions from the flap brackets. ${ }^{12}$ For FENoRFins (Fig. 9d), the agreement is good only up to about $5 \mathrm{kHz}$ before the device self-noise and bracket noise become dominant.

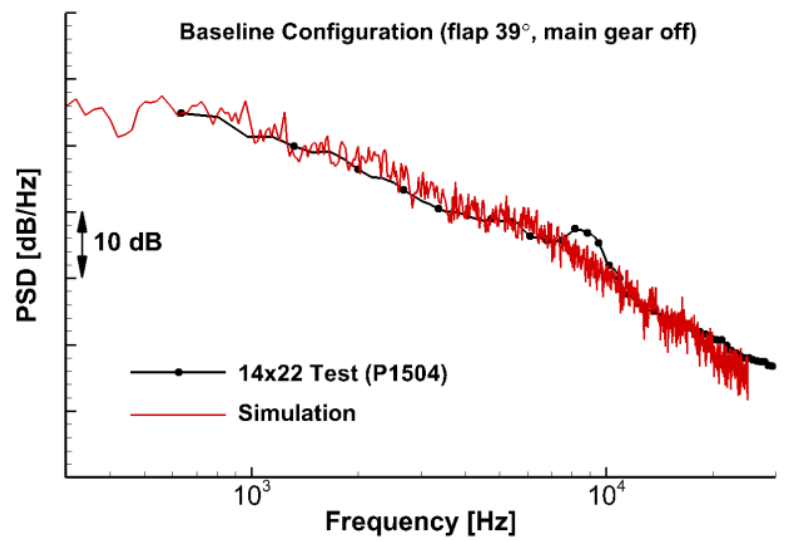

a) Baseline

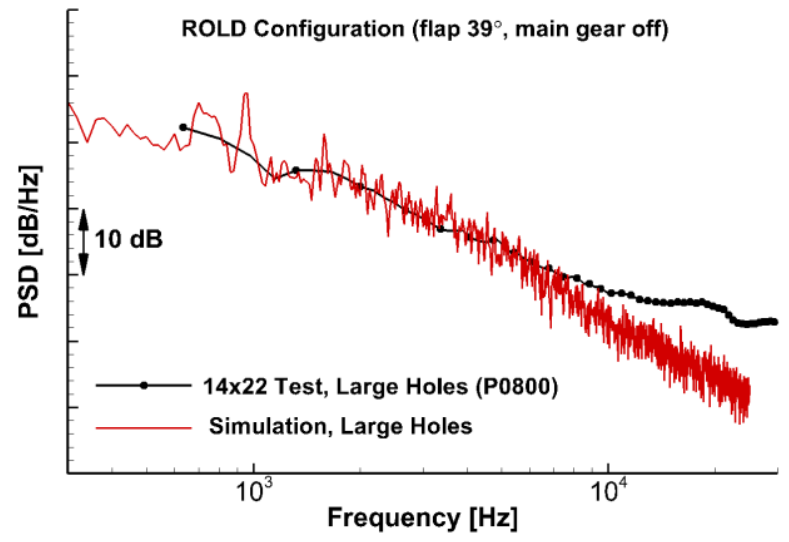

c) ROLD

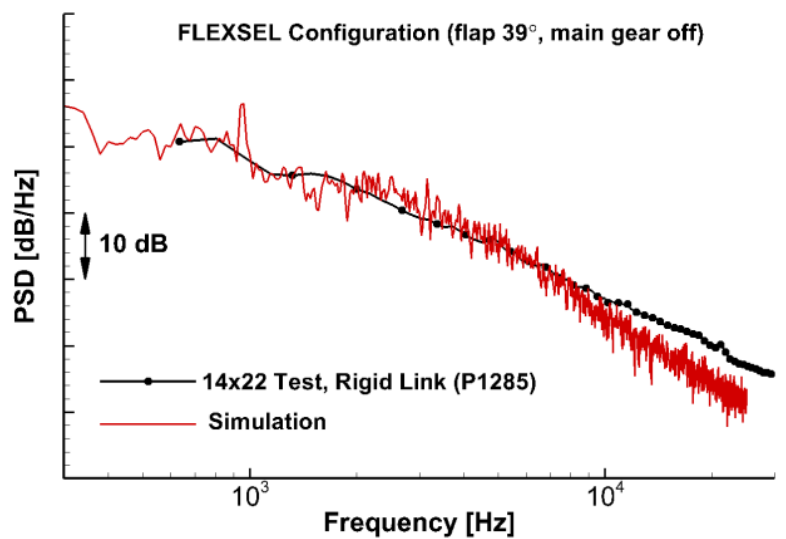

b) FLEXSEL

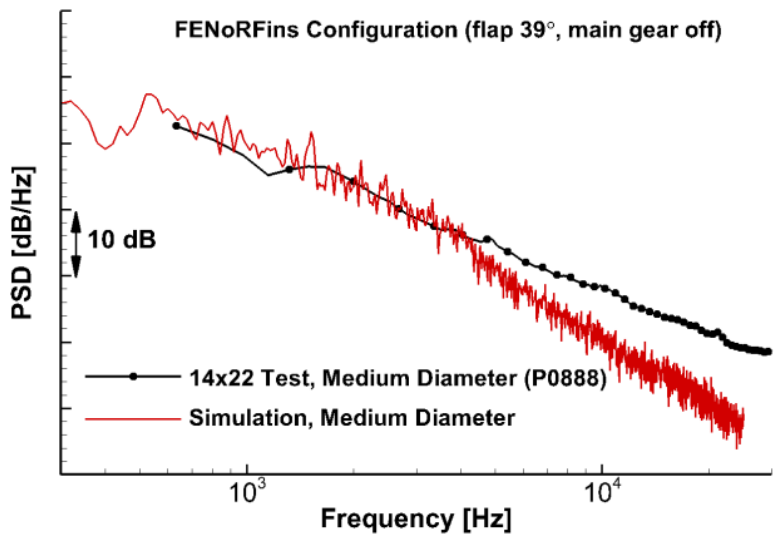

d) FENoRFins

Figure 9. Comparison between simulated and measured far field noise spectra 


\section{Main Landing Gear Fairings}

Several noise reduction concepts and fairings installed on the isolated $18 \%$ model scale main landing gear were simulated and compared to acoustic measurements that were conducted in the VA Tech Stability Tunnel during July of 2012 (unpublished results). The numerically predicted ranking of the gear concepts based on their acoustic performance was corroborated by the measurements and the farfield spectra were found to be generally in good agreement with the test results. Only one combination of the concepts applied to the isolated gear model is documented here. This combination provided the best overall noise reduction potential and was subsequently tested during the $14 \times 22$ test campaign of the semi-span model. ${ }^{12}$ Figure 10 shows the combination of the concepts that were collectively installed on the main landing gear. Additional details regarding these concepts are provided in Ref. 12.

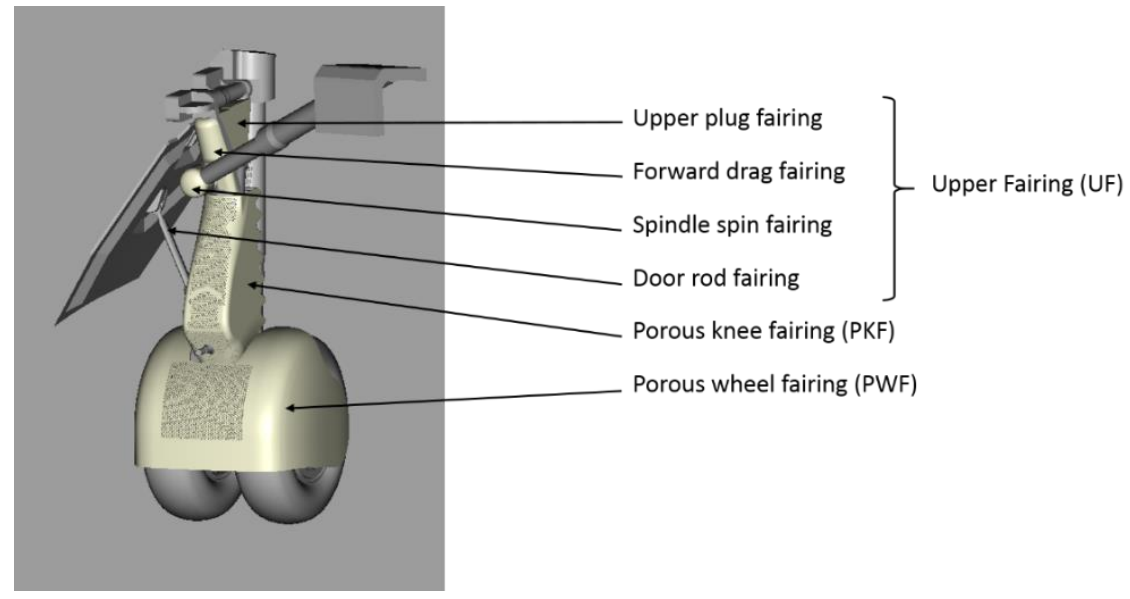

Figure 10. Noise reduction concepts for the main landing gear

The computed farfield spectrum for the baseline gear configuration and the corresponding measured spectrum from the VA Tech test at the overhead position of $2.27 \mathrm{~m}$ from the model are shown in Fig. 11. Notice that the spectra are displayed in full scale frequencies. Very good agreement between the two spectra up to the measured frequency of $2 \mathrm{kHz}$ is observed. The tonal peak at $\sim 460 \mathrm{~Hz}$ in the predicted noise is associated with the presence of an opening at the knee joint of the front strut. Because the front strut is hollow, passage of air through this opening causes the cavity to resonate. This resonance is clearly visualized in the band filtered fluctuating pressures displayed in Fig. 11. While the strut cavity is completely hollow in the simulated geometry, it was partially filled with pressure tubing and wires during the experiment. The difference in cavity volume may partially explain the frequency shift of this peak in the experiment.
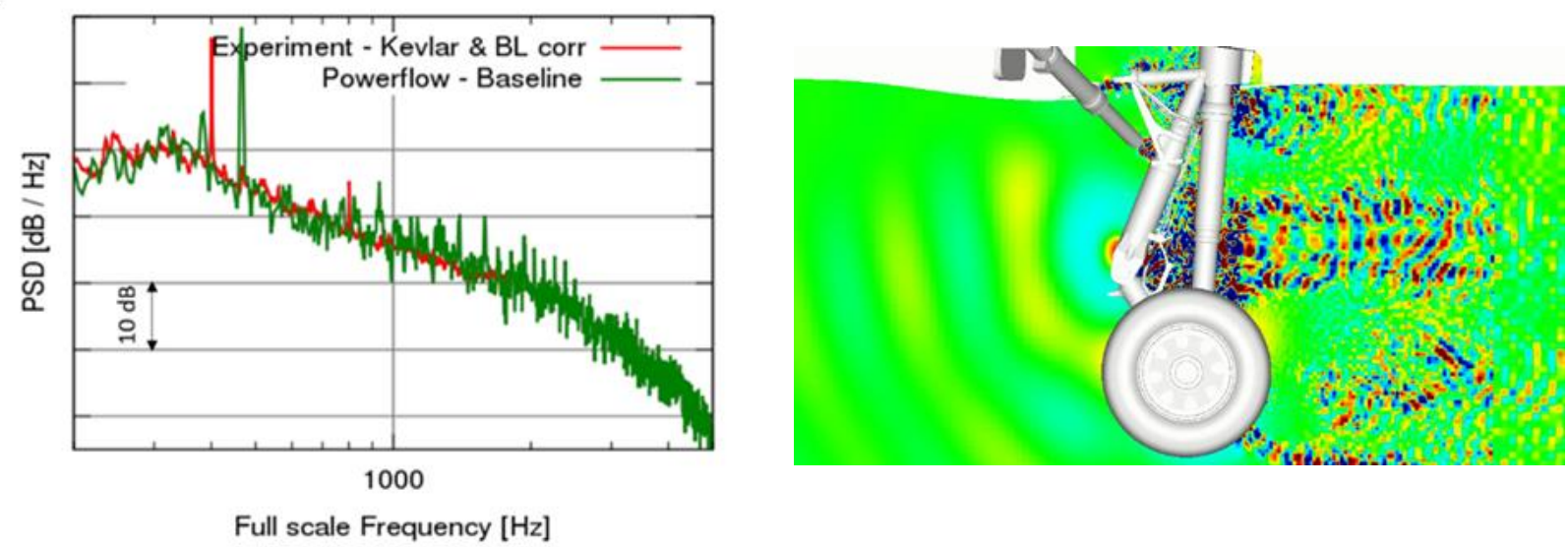

Figure 11. Far field noise comparison for the baseline main landing gear with measurement (left) and snapshot of the band filtered pressure field at $460 \mathrm{~Hz}$ sampled at the center plane of the main landing gear (right) 
A qualitative comparison of the simulated flow fields for the two configurations without and with the noise reduction concepts installed is provided in Fig. 12 and Fig. 13. Contours of the total pressure and acoustic dilation fields are given in Fig. 12 for a vertical plane bisecting the gear and in Fig. 13 for a horizontal plane cutting through the mid-section of the wheels. The immediate wake shows noticeable alteration of the flow field and increased pressure losses due to the presence of the gear concepts. The time derivative of the static pressure field (presented in the black and white figures on the right columns) clearly shows that the installed devices substantially reduce the amplitude of the pressure waves radiating from the gear.
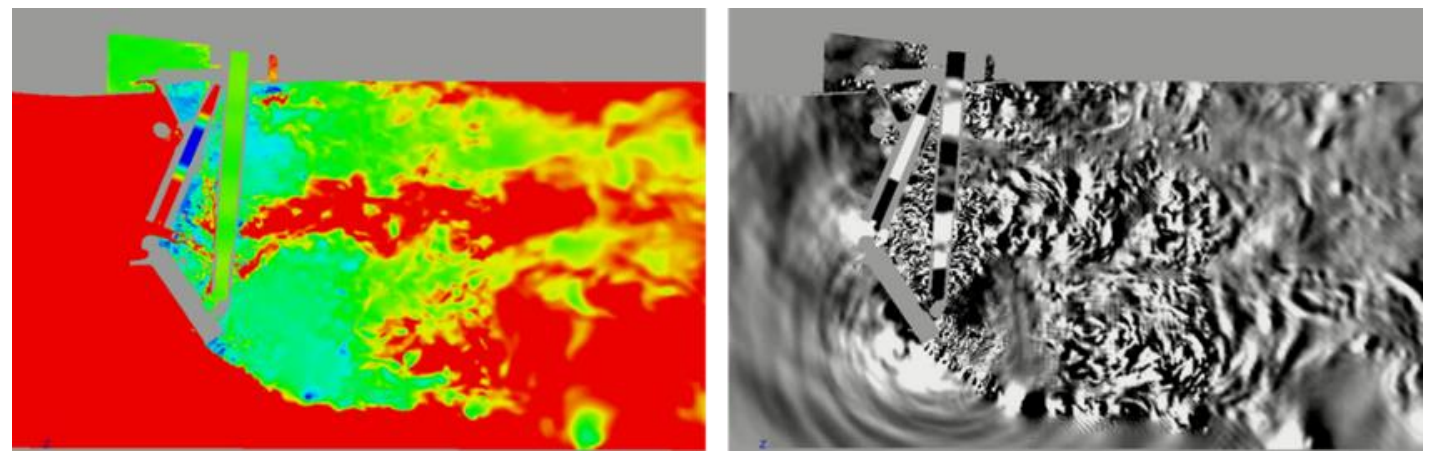

a) Without noise reduction concepts
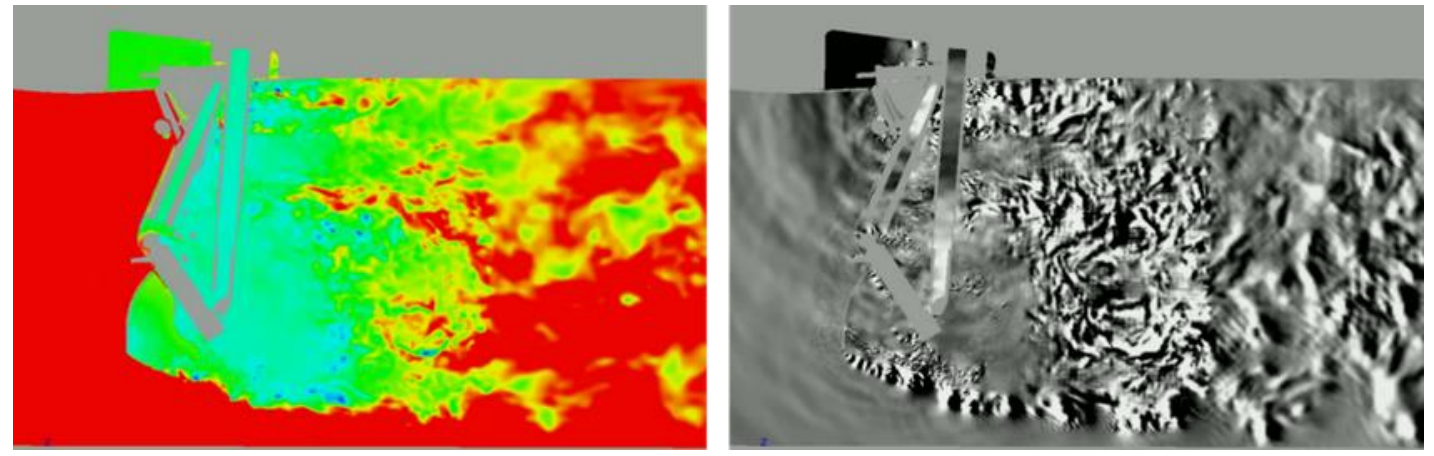

b) With noise reduction concepts

Figure 12. Snapshot of the total pressure field (left) and the acoustic dilatation field (right) in a plane cutting vertically though the center of the main landing gear wheels
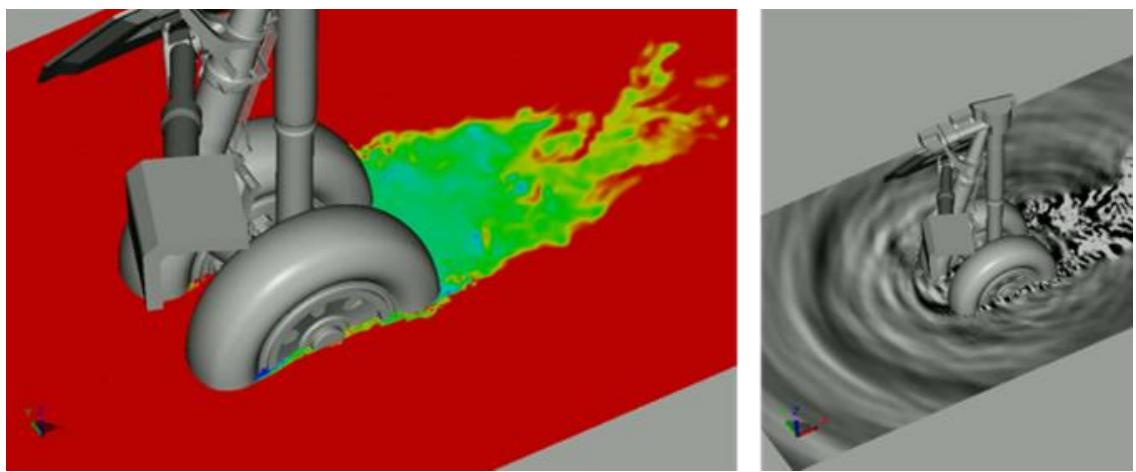

a) Without noise reduction concepts

Figure 13. Snapshot of the total pressure field (left) and the acoustic dilatation field (right) in a plane cutting horizontally though the center of the main landing gear wheels 

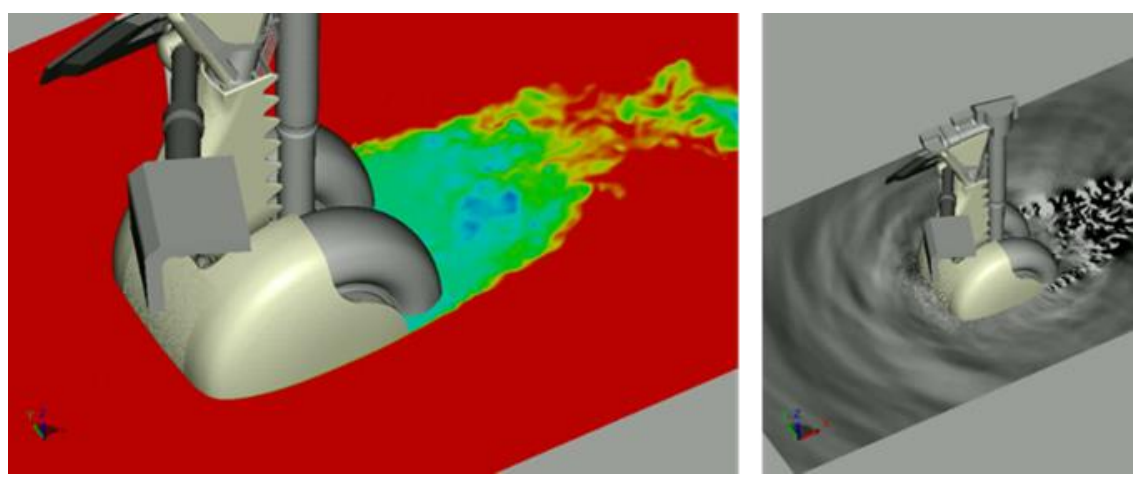

b) With noise reduction concepts

Figure 13. Concluded.

The corresponding farfield spectra for the two simulated configurations are plotted in Fig. 14. The spectra indicate that good noise reduction performance was obtained across a broad frequency range of about $5 \mathrm{kHz}$. Notice that introduction of the porous knee fairing results in cancellation of the prominent tonal peak associated with the hollow front strut. While removing this high amplitude tone would automatically yield significant reduction in the OASPL, the more difficult task of lowering the broadband component of gear noise is the ultimate goal of the present effort. Thus, to determine how effective this combination of gear concepts is, OASPLs were computed by performing the integration of the spectra over two different frequency ranges. The first frequency range starts at $500 \mathrm{~Hz}$ and hence beyond the tonal peak at $\sim 460 \mathrm{~Hz}$. The second frequency range starts at $200 \mathrm{~Hz}$ but excludes the tonal peak through manual smoothing of the spectrum. The results are listed in Table 3 . Both unweighted $(\mathrm{dB})$ and $\mathrm{D}$-weighted $(\mathrm{dBD})$ metrics confirm very promising noise reduction levels that are $\sim 4 \mathrm{~dB}$.

\section{Measured vs computed noise PSD at B-F array center}

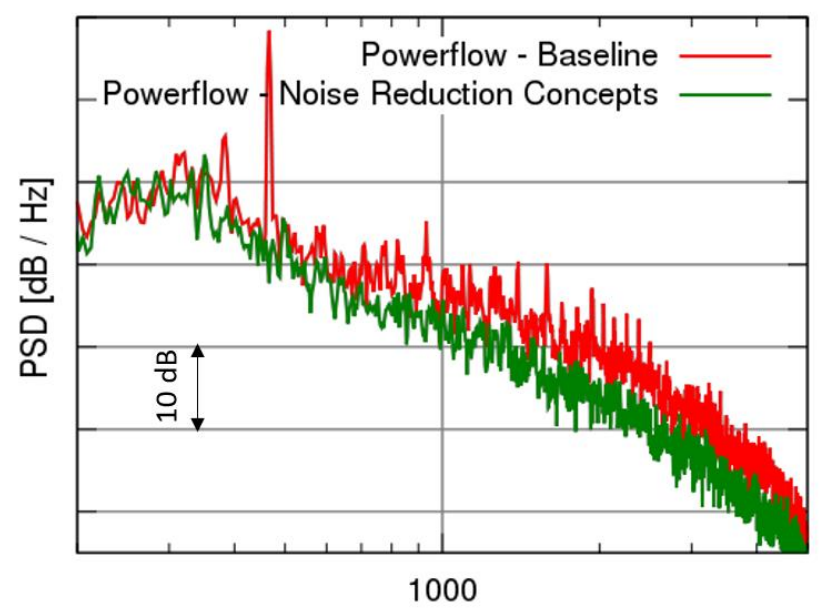

Full scale Frequency $[\mathrm{Hz}]$

Figure 14. Far field noise comparison for the baseline main landing gear with and without noise reduction concepts 
Table 3 Predicted noise reduction with and without tone in $\mathrm{dB}$ and $\mathrm{dBD}$ metrics

\begin{tabular}{|c|c|c|c|}
\hline $\begin{array}{c}\Delta \text { OASPL dB } \\
500 \mathrm{~Hz}-5000 \mathrm{~Hz}\end{array}$ & $\begin{array}{c}\Delta \text { OASPL dBD } \\
500 \mathrm{~Hz}-5000 \mathrm{~Hz}\end{array}$ & $\begin{array}{c}\Delta \text { OASPL dB } \\
200 \mathrm{~Hz}-5000 \mathrm{~Hz} \\
\text { (tone removed in } \\
\text { baseline) }\end{array}$ & $\begin{array}{c}\Delta \text { OASPL dBD } \\
200 \mathrm{~Hz}-5000 \mathrm{~Hz} \\
\text { (tone removed in } \\
\text { baseline) }\end{array}$ \\
\hline 4.3 & 4.7 & 3.9 & 4.3 \\
\hline
\end{tabular}

\section{Combined Flap-Side-Edge and Main Landing Gear Noise Reduction Concepts}

Simulations involving simultaneous evaluation of flap and gear concepts were conducted after the $201314 \times 22$ test campaign was completed. These simulations were performed as part of the down selection process on the noise reduction combinations that were under consideration for future flight testing. As such, most of the simulations involved configurations that were very similar, but not identical, to those evaluated during the $14 \times 22$ test. A quiet landing configuration involving concurrent treatment of flap and landing gear is shown in Fig. 3. For this configuration, the noise reduction concepts comprise the collection of main landing fairings summarized in Fig. 10 and the FENoRFins flap side edge concept displayed in Fig. 1. The predicted aerodynamic performance change due to the installed noise reduction concepts was within $0.3 \%$ of the performance for the baseline configuration, well within the uncertainty range of both computed and measured values. The predicted minimal impact to aerodynamic performance was corroborated by the test results. ${ }^{11}$ The complex three dimensional flow field shown in Fig. 15a illustrates the interaction between the main landing gear wake and the inboard flap side edge. Notice that the wake produced by the main landing gear fairing is somewhat larger and contains finer vortex filaments as compared to the baseline. A larger wake was also observed in the total pressure contours of the isolated gear configuration shown in Fig. 12. The larger wake and the additional finer flow structures do not necessarily translate into an increase in noise. As shown qualitatively in the dilatation field on the model surface (Fig. 15b), the baseline (untreated) configuration clearly illustrates the presence of high-amplitude radiating pressure waves emanating from the noise sources associated with the main landing gear and the inboard flap edge. The attenuation of the sound sources when the noise reduction concepts are applied is visible in the surface wave patterns for the quiet configuration.

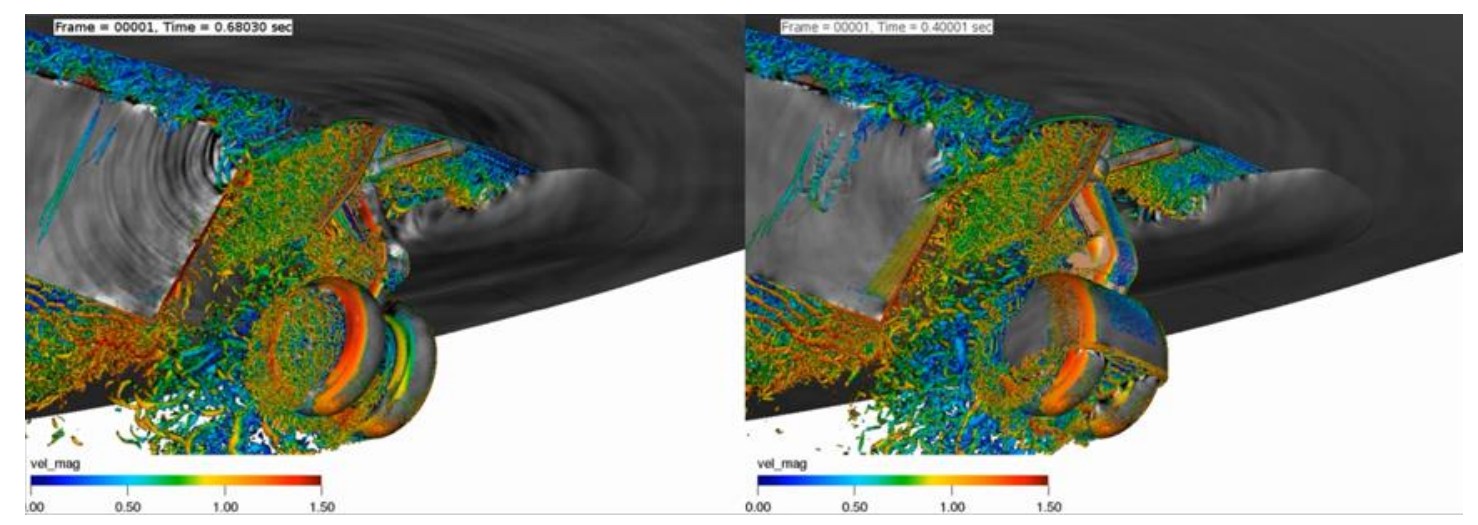

a) Isosurface of of $\lambda_{2}$ (colored by velocity magnitude)

Figure 15. Snapshot of the three dimensional flow field near the main landing gear and inboard flap side edge for the baseline (left) and low noise configuration (right) 


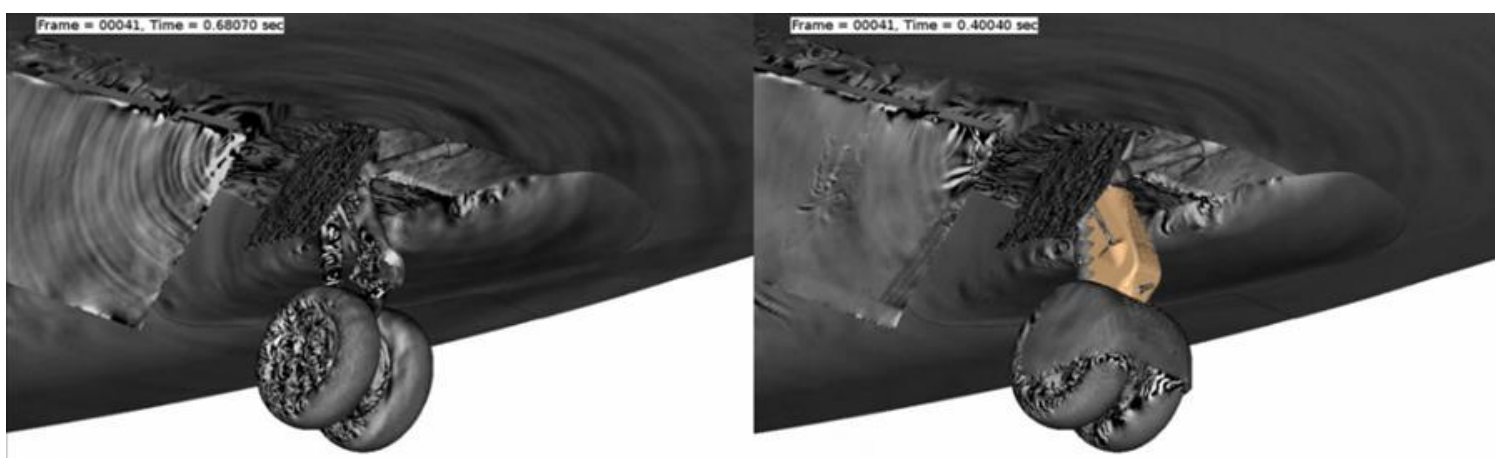

b) Pressure derivative field representing the acoustic dilatation on the surface

Figure 15. Concluded

Comparisons between computed and measured far field noise spectra obtained at the overhead location of $5.33 \mathrm{~m}$ from the model as installed in the $14 \times 22$ tunnel is presented in Fig. 16. The combination of flap and gear noise reduction concepts used in the measured data is the closest configuration to that used in the simulation. In addition to the combination of flap and gear concepts that were considered in the simulation, the tested configuration included a gear cavity treatment consisting of a stretchable mesh applied to the cavity opening (see Ref. 12 for more details). Also, the porous area on the frontal face of the wheel fairing was taped over in the test. The experiments ${ }^{12}$ demonstrate that 1) the cavity treatment reduced noise by $1-2 \mathrm{~dB}$ at frequencies below $5 \mathrm{kHz}$, and 2) porous and non-porous versions of the wheel fairing provided nearly the same amount of noise reduction.

Observe from Fig. 16 that the predicted spectra track the measured spectra both in level and frequency content. For the treated case (Fig. 16b), the slight over-prediction of noise at frequencies below $4 \mathrm{kHz}$ could be attributed to the missing cavity treatment in the simulated configuration. Starting at $15 \mathrm{kHz}$, the computed spectrum levels gradually fall below measured data. The higher noise levels in the measured spectrum are attributed to FENoRFins self-generated noise, which was not captured by the simulation. Nevertheless, given the extreme geometrical complexities of the simulated configuration, the comparison is quite satisfactory. The predicted overall noise reduction at the overhead microphone position is $3.3 \mathrm{~dB}(3.9 \mathrm{dBD})$ including the few prominent tones observed in the simulated baseline spectrum of Fig. 16a, and $2.7 \mathrm{~dB}(3.6 \mathrm{dBD})$ with the tonal peaks removed. A directivity pattern for angles $30^{\circ}$ through $120^{\circ}\left(30^{\circ}\right.$ forward, $90^{\circ}$ overhead, $120^{\circ}$ rearward) is depicted in Fig. 17 in the perceived noise level (PNL) metric $^{18}$ for both configurations. These patterns indicate that 1) the untreated configuration generates noise with significant tonal content, as manifested by the appearance of directivity lobes, 2) forward radiation is dominant, and 3) substantial noise reduction was achieved for a wide flyover angle range by treating the flap side edge and the main landing gear. This represents a very promising broadband noise reduction for the realistic complete configuration.

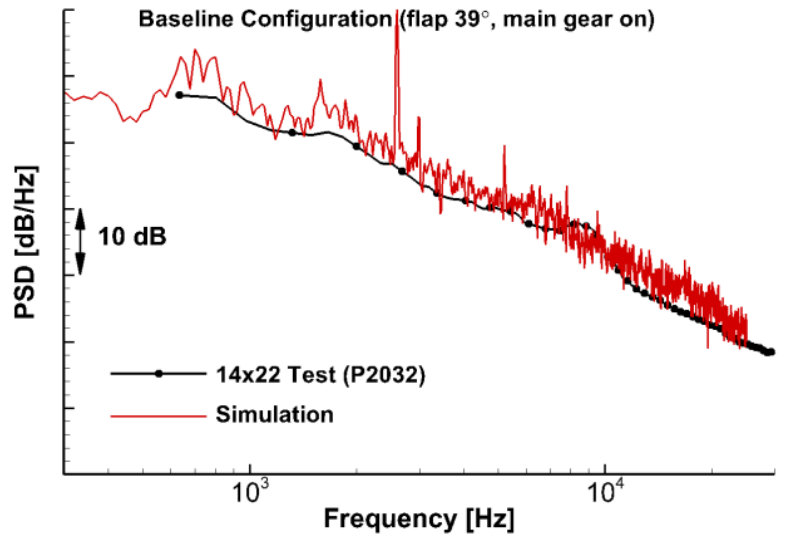

a) Baseline

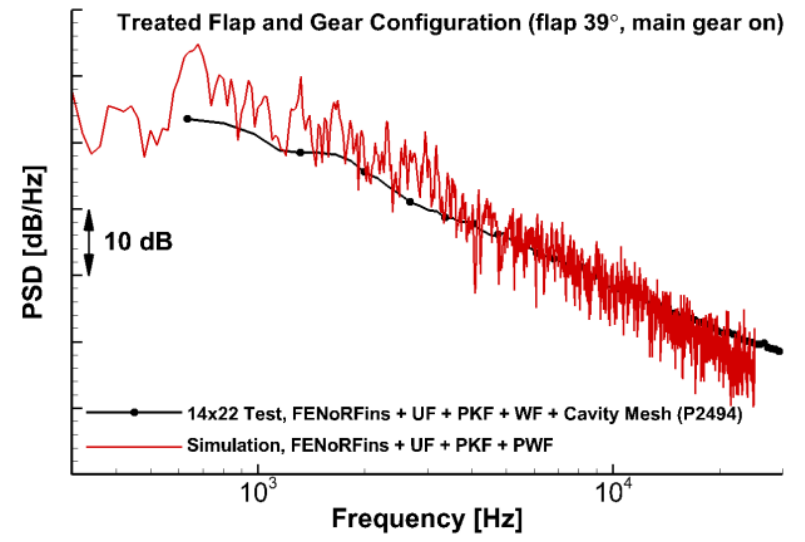

b) Low noise

Figure 16. Comparison between simulated and measured far field noise spectra for baseline configuration and low noise configuration 


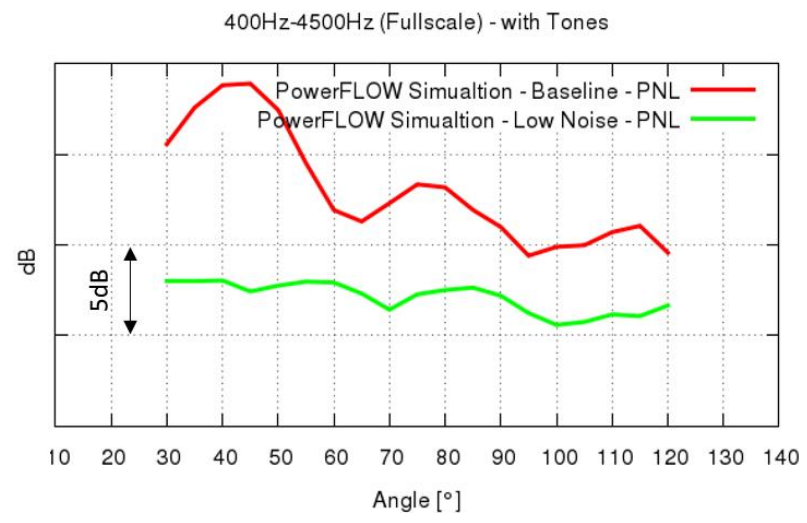

a) With tonal content

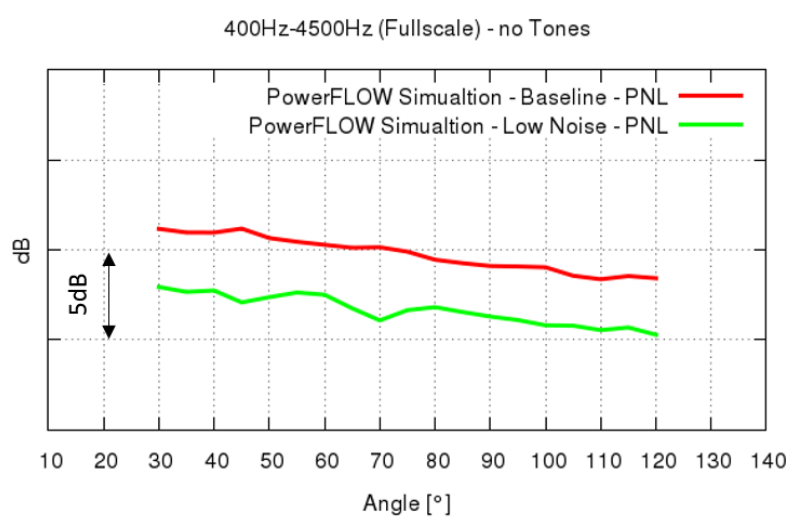

b) Without tonal content

Figure 17. Predicted directivity in PNL for baseline and low noise configurations

\section{Concluding Remarks and Outlook}

A hybrid lattice Boltzmann and Ffowcs Williams and Hawkings approach was used to perform extensive flow and aeroacoustic simulations of a complex aircraft model. The simulated geometry corresponds to a high fidelity, $18 \%$ scale, semi-span model that was subsequently tested in the NASA LaRC 14x22 foot subsonic tunnel. The pre-test computational effort targeted 1) prediction of farfield noise associated with the model prominent airframe sources, namely, the deflected-flap tips and main landing gear; 2) evaluation and refinement of various flap and main landing gear noise reduction concepts, and 3) identification of potential improvements to the noise reduction designs. In this paper, results from the pre-test simulations were compared to experimental data.

In general, the comparisons were very promising and highlighted the maturity of the underlying numerical approach to correctly capture the relevant airframe noise generation mechanisms and provide the necessary insight for a proper ranking of the best performing noise reduction concepts, some having very complex geometrical detail. The comparisons also indicated that some of the high frequency discrepancies observed between predicted and measured data could be associated with insufficient spatial resolution in the simulation.

The current approach is being extended to a full scale, full aircraft geometry in landing configuration. The simulations for the baseline geometry will be used to highlight Reynolds number and geometrical fidelity effects on steady and unsteady surface pressures, off-surface flow field, and farfield acoustics. In addition, several of the best performing noise reduction concepts numerically evaluated on the $18 \%$ scale model will be scaled up and their performance evaluated at Reynolds numbers similar to those encountered by the full scale aircraft during landing.

\section{Acknowledgments}

This work was supported by the Environmentally Responsible Aviation (ERA) project under the Integrated System Research Program (ISRP) of NASA. The authors would like to thank Enrico Fabiano and Bruno Moschetta for simulation geometry preparation of the noise reduction concepts. Our gratitude is extended also to Domenica Fresca and Patrick Moran for the complex three dimensional post processing of the data and generation of the animations.

\section{References}

1 Dobrzynski, W., “Almost 40 Years of Airframe Noise Research: What Did We Achieve," J. Aircraft, Vol. 47, No.2, pp. 353-367, 2010.

2 Fink, M., "Noise Component Method for Airframe Noise," J. Aircraft, Vol. 16, No.10 pp. 659-665, 1979.

3 Guo, Y., Yamamoto, K .J., and Stoker, R. W., "Component-Based Empirical Model for High-Lift System Noise Prediction," J. Aircraft, Vol. 40, No.5, pp. 914-922, 2003.

4 Rackl, R. G., Miller, G., Guo, Y., and Yamamoto, K., "Airframe Noise Studies - Review and Future Directions," NASA Technical Report NASA/CR-2005-213767, June 2005.

5 https://info.aiaa.org/tac/ASG/FDTC/DG/BECAN_files_/BANCIII.htm, visited on 16 ${ }^{\text {th }}$ October 2014.

6 Lockard, D. "Summary of the Tandem Cylinder Solutions from the Benchmark problems for Airframe Noise Computations-I Workshop," AIAA Paper 2011-0353, 2011. 
7 Khorrami, M. R. and Mineck, R. E., "Towards Full Aircraft Airframe Noise Prediction: Detached Eddy Simulations," AIAA Paper 2014-2480, 2014.

8 Khorrami, M. R., Fares, E., and Casalino, D., "Towards Full-Aircraft Airframe Noise Prediction: Lattice-Boltzmann Simulations," AIAA Paper 2014-2481, 2014.

9 Khorrami, M. R., Hannon, J. A., Neuhart, D. H., Markowski, G. A., and Van de Ven, T., "Aeroacoustic Studies of a High-Fidelity Aircraft Model: Part 1-Steady Aerodynamic Measurements," AIAA Paper 2012-2233, 2012.

${ }^{10}$ Khorrami, M. R. and Neuhart, D. H, "Aeroacoustic Studies of a High-Fidelity Aircraft Model: Part 2- Unsteady Surface Pressures," AIAA Paper 2012-2234, 2012.

${ }^{11}$ Neuhart, D., Hannon, J., and Khorrami, M. R., "Aerodynamic Measurements of a Gulfstream Aircraft Model with and without Noise Reduction Concepts," AIAA Paper 2014-2477, 2014.

${ }^{12}$ Khorrami, M. R., Humphreys, W. M.Jr., Lockard, D. P., and Ravetta, P. A., "Aeroacoustic Evaluaion of Flap and Landing Gear Reduction Concepts," AIAA Paper 2014-2478, 2014.

${ }^{13}$ Chen, H., "Volumetric Formulation of the Lattice-Boltzmann Method for Fluid Dynamics: Basic Concept," Physical Review E, Vol. 58, No. 3, pp. 3955-3963, 1998.

${ }^{14}$ Chen, H., Texeira, C., and Molvig, K., "Realization of Fluid Boundary Condition via Discrete Boltzmann Dynamics," Int. Journal of Modern Physics C, Vol.09, pp. 1281-1292, 1998.

${ }^{15}$ Chen, H., Kandasamy, S., Orszag, S., Shock, R., Succi, S., and Yakhot, V., "Extended Boltzmann Kinetic Equation for Turbulent Flows," Science, No. 301, pp. 633-636, 2003.

${ }^{16}$ Chen, S., and Doolen, G. D., "Lattice Boltzmann Method for Fluid Flows," Annual Review of Fluid Mechanics, Vol. 30, pp 329-364, 1998.

${ }^{17}$ Fares, E. and Noelting, S., "Unsteady Flow Simulation of a High-Lift Configuration using a Lattice-Boltzmann Approach," AIAA Paper 2011-0869, 2011.

${ }^{18}$ Casalino, D., Noelting, S., Fares, E., Van de Ven, T., Perot, F., and Bres, G., "Towards Numerical Aircraft Noise Certification: Analysis of a Full-Scale Landing Gear in Fly-Over Configuration," AIAA Paper 2012-2235, 2012.

${ }^{19}$ Casalino D., et al., "Towards Lattice Boltzmann Predictions of Turbofan Noise," AIAA Paper 2014-3101, 2014.

${ }^{20}$ Chen, H., Chen, S., and Matthaeus, W., "Recovery of the Navier-Stokes Equations Using a Lattice-Gas Boltzmann Method," Physical Review A, Vol. 45, No. 8, pp. 5339-5342, 1992.

${ }^{21}$ Qian Y. H., D'Humières, D., and Lallemand, P., "Lattice BGK Models for Navier-Stokes Equation," Europhysics Letters, Vol. 17, pp. 479-484, 1992.

${ }^{22}$ Marié, S., Ricot, D., and Sagaut, P., "Comparison between lattice Boltzmann method and Navier-Stokes high order schemes for computational aeroacoustics," J. of Computational Physics, Vol. 228, pp. 1056-1070, 2009.

${ }^{23}$ Brès, G. A., Pérot, F., and Freed, D. "Properties of the Lattice-Boltzmann Method for Acoustics," AIAA Paper 2009-3395, May 2009.

${ }^{24}$ Shan, X.,Yuan, X. F., and Chen, H., "Kinetic theory representation of hydrodynamics: a way beyond the NavierStokes equation," J. of Fluid Mechanics, Vol. 550, pp 413- 441, 2006.

${ }^{25}$ Zhang, R., Shan, X., and Chen, H., "Efficient kinetic method for fluid simulation beyond the Navier-Stokes equation," Physical Review E, Vol. 74, p. 046703, 2006.

${ }^{26}$ Chen, H., Kandasamy, S., Orszag, S. A., Succi, S., and Yakhot, V., "Extended Bolzmann Kinetic Equation for Turbulent Flows," Science, Vol. 301, No. 5633, pp. 633-636, 2003.

${ }^{27}$ Yakhot, V. and Orszag, S. A., "Renormalization Group Analysis of Turbulence. I. Basic Theory," J. Sci. Comput., Vol 1, No. 2, pp. 3-51, 1986.

${ }^{28}$ Menter, F., Kuntz, M., and Bender, R., "A Scale Adaptive Simulation Model for Turbulent Flow Predictions," AIAA Paper 2003-0767, 2003.

${ }^{29}$ Chen, H., Orszag, S., Staroselsky, I., and Succi, S. "Expanded Analogy between Boltzmann Kinetic Theory of Fluid and Turbulence," J. of Fluid Mechanics, Vol. 519, pp. 307-314, 2004.

${ }^{30}$ Fares, E. "Unsteady Flow Simulation of the Ahmed Reference Body using a Lattice Boltzmann Approach," J. of Computers and Fluids, No. 35, pp. 940-950, 2006.

${ }^{31}$ König, B., Fares, E., Noelting, S., Jammalamadaka, A., and Li, Y., "Investigation of the NACA 4412 Trailing Edge Separation using a Lattice-Boltzmann Approach," AIAA Paper 2014-3324, 2014.

${ }^{32}$ Ffowcs Williams, J. E. and Hawkings, D. L., "Sound Generated by Turbulence and Surfaces in Arbitrary Motion," Philosophical Transactions of the Royal Society, Vol. A264, No. 1151, pp. 321-342, 1969.

${ }^{33}$ Farassat, F. and Succi, G. P., "The Prediction of Helicopter Discrete Frequency Noise," Vertica, Vol. 7, No. 4, pp. 309-320, 1983.

${ }^{34}$ Najafi-Yazdi, A., Brès, G. A., and Mongeau, L., "An Acoustic Analogy Formulation for Moving Sources in Uniformly Moving Media," Proceeding of The Royal Society of London A, Vol. 467 (2125), pp. 144-165, 2011. 
${ }^{35}$ Khorrami, M. R., Lockard, D. P., Humphreys, Jr., W. M., Choudhari, M. M., and Van de Ven, T., "Preliminary Analysis of Acoustic Measurements from the NASA-Gulfstream Airframe Noise Flight Test," AIAA Paper 20082814, May 2008.

${ }^{36}$ Casalino, D., Fares, E., Duda, B., Hazir, A., and Khorrami, M. R., "On the Connection between Flap Side-Edge Noise and Tip Vortex Dynamics," to be presented at the Aviation Conference 2015, AIAA Paper 2015, Dallas. 